Chir Gastroenterol 1997;13(suppl 2):I-III

\title{
Inhalt - Contents, Vol. 13, Supplement 2, 1997
}

Gründungsherausgeber
A. Akovbiantz $\dagger$, Zurich
H. Denck, Wien
K.-J. Paquet, Hannover
C. E. Zöckler, Bad Oeynhausen
Hauptschriftleiter
K.-J. Paquet, Hannover
Schriftleitung
Ch. E. Broelsch, Hamburg
M. W. Büchler, Bern
J. Horn, München
H. Säuberli, Baden
R. Schiessel, Wien
Assistenz:
K. Glaser, Innsbruck
J. R. Izbicki, Hamburg
E. Klar, Heidelberg
Bibliographischer Hinweis:

Die Zeitschrift ist in der Excerpta-Medica-Datenbank EMBASE indexiert.

Wissenschaftlicher Beirat

Deutschsprachiger Raum

H. M. Becker, München M. Betzler, Essen E. Bodner, Innsbruck H. Bosseckert, Jena H. Bülow, Schweinfurt R. Dölp, Fulda K. E. Frede, Basel

D. Fuchs, Innsbruck

A. Gangl, Wien

J. M. Hackl, Innsbruck

E. G. Hahn, Erlangen/Nürnberg

J. Hauss, Leipzig

P. Hermanek, Erlangen/Nürnberg W. Hohenberger, Erlangen/Nürnberg S. Liebe, Rostock H.

Lippert, Magdeburg

Ausland

J. Alexander-Williams, Birmingham

J. Gallinger, Moskau

J. M. Henderson, Cleveland

R. A. Hinder, Omaha

Y. Idezuki, Tokyo

I. Ihse, Lund

C. W Imrie, Glasgow

G. G. Jamieson, Adelaide 


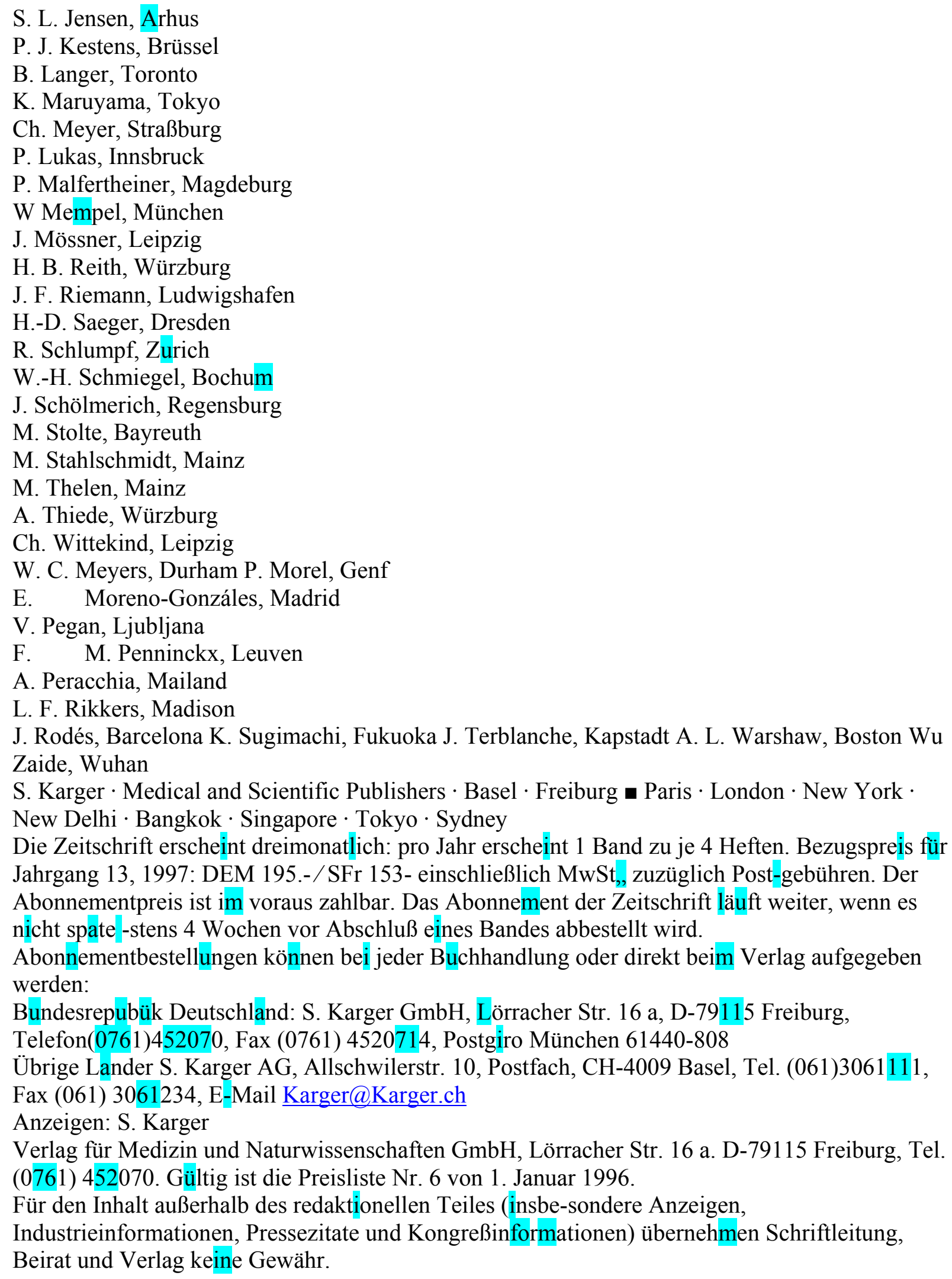


Eine Markenbezeichnung kann warenzeichenrechtlich ge-schützt sein, auch wenn bei ihrer Verwendung in dieser Zeitschrift das Zeichen ${ }^{\circledR}$ oder ein anderer Hinweis auf etwa bestehende Schutzrechte fehlen sollte. Für Satzfehler, insbe-sondere bei Dosierungsangaben, wird keine Gewähr über-nommen.

Die Zeitschritt sowie alle in ihr enthaltenen einzelnen Beiträge und Abbildungen sind urheberrechtlich geschützt. Jede Ver-wertung, die nicht ausdrücklich vom Urheberrechtsgesetz zu-gelassen ist, bedarf der vorherigen Zustimmung des Verlags. Das gilt insbesondere für Vervielfältigung, Bearbeitungen, Übersetzungen, Mikroverfilmungen und die Einspeicherung und Verarbeitung in elektronischen Systemen.

Supplement 2 zu Band 13,

Dezember 1997.

ISBN 3-8055-6635-2

Ladenpreis des Sonderheftes:

DEM 40,- inkl. MwSt.

Abonnenten erhalten es unberechnet

im Rahmen des Abonnements.

(C) Copyright 1997 by S. Karger

Verlag für Medizin und Naturwissenschaften $\mathrm{GmbH}$

Lörracher Straße 16 a, D-79115 Freiburg

Verlagsleitung und presserechtlich verantwortlich: Sibylle Hopf

Redaktionsassistenz: Dr. Hannelore Tenckhoff Anzeigenverwaltung: Christiane Opitz

Produktionsleitung: Georg Brunner

Herstellung: druckhaus köthen GmbH Friedrichstral11/8 11/12, D-06366 Köthen/Anhalt

\section{KARGER}

(C) 1997 S. Karger GmbH, Freiburg Fax (0761) 4520714 http://www.karger.ch

Chirurgiscl-ie

Inhalt - Contents

it interdisztplíiären Gesprächen f

Band 13, Supplement 2, Dezember 1997

Vol. 13, Supplement 2, December 1997

Inhalt

Contents

\section{Editorial}

Franke, S.; Reith, H. B. (Würzburg)

1 Konservative Therapie bei arterieller Verschlußkrankheit Stadium III/IV - eine

Standortbestimmung

Jeßberger, S.; Reith, H. B.; Debus, E. S.; Schmidt, K.; Franke, S. (Würzburg)

6 Femorokrurale Rekonstruktionen

Franke, S.; Gießel, I. (Würzburg)

12 Angiographie der kruropedalen Ausflußbahn -Kommentar aus Sicht des Radiologen

Schindler, G.; Schultheiss, D. (Würzburg)

15 Der Fibularisbypass - Analyse der Patientendaten der Universität Würzburg zwischen 1990 und 1994 
Timmermann, W; Gießel, I.; Deppisch, S.; Gassel, H.-J.; Franke, S. (Würzburg)

19 Interventionelle Techniken in der kruralen Gefäßchirurgie: PTA, ITA und Laser

Ebner, H.; Mani, G; Bonatti, G. (Bozen)

25 Lumbale Sympathektomie/Sympathikolyse in der Therapie der arteriellen Verschlußkrankheit

- Kommentar

Mittelkötter, U. (Würzburg); Kozuschek, W. (Bochum)

27 Der embolische Verschluß der unteren Extremität -femoraler, trunkaler und pedaler Zugang

Debus, E. S.; Reith, H. B.; Jeßberger, S.; Franke, S.; Thiede, A. (Würzburg)

31 Bypassmaterialien in der kruralen Chirurgie -eine Standortbestimmung

Freys, S. M. (Würzburg)

40 Postoperative Komplikationen nach rekonstruktiven

Eingriffen am arteriellen System - Prophylaxe und Handling

Reith, H. B.; Franke, S. (Würzburg)

II Impressum

U2 Hinweise für Autoren (2. Umschlagseite)

IV Editorial

Franke, S.; Reith, H. B. (Würzburg)

1 Conservative Therapy in Patients with Peripheral Arterial Occlusive Disease Stage III/IV -

State of the Art

Jeßberger, S.; Reith, H. B.; Debus, E. S.; Schmidt, K.; Franke, S. (Würzburg)

6 Femoro-crural Reconstructions

Franke, S.; Gießel, I. (Würzburg)

12 Angiography of the Cruropedal 'Run-Off' Pathway -Commentary of the Radiologist

Schindler, G; Schultheiss, D. (Würzburg)

15 Peroneal Artery Bypass - Analysis of Patient Data at the University of Würzburg, 1990-1994

Timmermann, W.; Gießel, I.; Deppisch, S.; Gassel, H.-J; Franke, S. (Würzburg)

19 Interventional Techniques in Crural Vessel Obstruction: PTA, ITA and Laser

Ebner, H.; Mani, G; Bonatti, G (Bozen)

25 Lumbar Sympathectomy/Sympathicolysis in the Therapy of Arterial Occlusive Disease -

Commentary

Mittelkötter, U. (Würzburg); Kozuschek, W. (Bochum)

27 Arterial Embolism of the Lower Limb -Femoral, Truncal, and Pedal Incisions

Debus, E. S.; Reith, H. B.; Jeßberger, S.; Franke, S.; Thiede, A. (Würzburg)

31 Bypass Material in Surgery - State of the Art

Freys, S. M. (Würzburg)

40 Postoperative Complications after Surgery of the Arteries Prophylaxis and Handling

Reith, H. B.; Franke, S. (Würzburg)

II Imprint

U3 Instructions to Authors (Inside back cover)

KAHGER

(C) 1997 S. Karger GmbH, Freiburg Fax (0761) 4520714 http://www.karger.ch

III

Chir Gastroenterol 1997;13(suppl 2):IV

EDITORIAL

S. Franke 


\section{H. B. Reith, Würzburg}

Nach dem französischen Angiologen Rene Fontaine (1899-1979) läßt sich die peri-phere arterielle Verschlußkrankheit (pAVK) der unteren Extremität in die Sta-dien I-IV unterteilen. Von klinischer Be-deutung für den Gefäßchirurgen sind das Stadium II, die eingeschränkte Gehstrecke - das Leitsymptom der pAVK -, vor allem jedoch das Stadium III, der zeitweilig oder dauernd auftretende Ruheschmerz, und das Stadium IV, die trockene Zehennekro-se oder feuchte Zehengangrän. Zum Stadium IV werden außerdem Hautulzera, insbesondere im Bereich der Ferse, und trophische Hautveränderungen im Bereich der Zehenkuppen mit Übergang auf den Vorfuß gerechnet. Klarheit schaffen die dopplersonographische Messung der Verschlußdrücke über der Arteria tibialis posterior und Arteria dorsalis pedis nach Belastung und die angiographische Abklä-rung.

Problematisch kann sich im Stadium II die klinische Situation gestalten, wenn angio-graphisch neben einem Verschlußprozeß der Arteria femoralis superficialis zusätz-lich eine

Abgangsstenose der Arteria pro-funda femoris vorliegt. Kommt es im Rahmen einer Progredienz des Grundlei-dens zu einer raschen Zunahme der Abgangsstenose im Bereich der A. profunda femoris, kann sich in kurzer Zeit - nicht selten innerhalb von wenigen Tagen - das Stadium II in ein Stadium IV umwan-deln.

Prinzipiell gilt, daß die Indikation für ei-nen kruralen Bypass gegeben ist, wenn bei dem gefäßkranken Patienten ein Stadium III oder IV vorliegt. Im Stadium III ist zu beachten, daß schon der diskrete, wäh-rend der Nacht auftretende Schmerz bei einem sonst tagsüber noch «normal» geh-fähigen älteren Gefäßpatienten ein deutli-

cher Hinweis sein kann und einer angiographischen Abklärung bedarf. Typi-scherweise wird von solchen Patienten anamnestisch mitgeteilt, daß dieser diskrete Schmerz beim Aufstehen oder beim Heraushängen des Beines aus dem Bett nachläßt und vollständig verschwindet. Bei Vorliegen einer feuchten Zehengangrän stellt sich die Frage, ob zunächst eine konservative antibiotische Therapie mit anschließender Minoramputation erfolgen sollte. Da beim Gefäßpatienten die Man-geldurchblutung eine Ursache für das Auf-treten einer Superinfektion im Sinne einer feuchten Gangrän darstellt, wird in einer operativen Sitzung zuerst revaskularisiert und anschließend zehen- oder vorfuß-amputiert.

Wie oben erwähnt, ist das Stadium II prinzipiell keine Indikation für die Implantation eines kruralen Bypasses, wobei aber im Stadium der «stark verminderten Geh-strecke» diese Frage je nach Situation indi-viduell abgeschätzt und beantwortet werden muß. Bei Vorliegen einer Abbruchgehstrecke von nur wenigen Metern auf dem standardisierten Laufband ist nach Ausschöpfung aller Möglichkeiten, wie absolutes Rauchverbot oder korrekte medikamentöse Einstellung vorliegender Risikofaktoren (koronare Herzkrankheit, Hypertonie, Diabetes mellitus, Hyper-cholesterinämie), im Einzelfall doch die Indikation für die Implantation eines kruralen Bypasses gegeben, insbesondere bei noch relativ jungen und «mitten im Leben stehenden» Gefäßpatienten. Im vorliegenden Heft soil dem Leser ein aktueller Stand zu den Möglichkeiten und Alternativen der kruralen Gefäßchirurgie inklusive der konservativen und interven-tionellen Therapie gegeben werden.

\section{KARGEIV}

(C) 1997 S. Karger GmbH, Freiburg Fax (07 61) 4520714 http://www.karger.ch

Prof. Dr. S. Franke, PD Dr. H. B. Reith Abteilung Gefäßchirurgie Chirurgische Klinik und Poliklinik Universität Würzburg Josef-Schneider-Straße 2 D-97080 Würzburg 